\title{
Infestação por triatomíneos em assentamentos e reassentamentos rurais na Região do Pontal do Paranapanema, Estado de São Paulo
}

\author{
Infestation by triatomines in rural settlement and resettlement areas \\ the Region of Pontal do Paranapanema, State of São Paulo
}

\author{
Rubens Antonio da Silva ${ }^{1}$, Susy Mary Perpétuo Sampaio ${ }^{2}$, Paulo Hiroshi Koyanagui ${ }^{2}$, \\ Marisa Poloni $^{2}$, Maria Esther de Carvalho ${ }^{1}$ e Vera Lúcia Cortiço Corrêa Rodrigues ${ }^{3}$
}

\begin{abstract}
RESUMO
Este estudo teve o objetivo de avaliar, em assentamentos e reassentamentos rurais, tendo em conta seu tempo de formação, características da infestação de habitações por triatomíneos. Analisamos dados referentes a 48 pesquisas de triatomíneos, colhidos durante o período entre janeiro de 1984 e junho de 2005, em 105 assentamentos e seis reassentamentos do Pontal do Paranapanema. Das 16 pesquisas realizadas nos assentamentos, sete $(43,8 \%)$ resultaram positivas. Todos os assentamentos positivos haviam sido estabelecidos oito ou mais anos antes. Das 32 pesquisas realizadas nos reassentamentos, 23 (71,9\%) resultaram positivas; todos os reassentamentos positivos haviam sido estabelecidos menos de oito anos antes. Uma vez que os habitantes dessas comunidades deslocam-se freqüentemente, nunca é demais salientar a necessidade de vigilância constante para detectar possíveis instalações de triatomíneos vetores em novos assentamentos.
\end{abstract}

Palavras-chaves: Doença de Chagas. Infestação por triatomíneos. Assentamentos rurais. Prevenção e controle.

\begin{abstract}
This study had the aim of assessing the characteristics of triatomine infestation in human dwellings in rural settlement and resettlement areas with regard to the time when infestation began. We analyzed data relating to 48 triatomine surveys carried out in 105 settlement areas and six resettlement areas in the region of Pontal do Paranapanema between January 1984 and June 2005. Among the 16 surveys in settlement areas, seven (43.8\%) had positive findings, all of them in communities established eight or more years previously. Among the 32 surveys in resettlement areas, 23 (71.9\%) had positive findings, all of them in communities established for periods shorter than eight years. Since the inhabitants of such communities frequently move, the need for constant vigilance to detect any cases of infestation by vector triatomines in new settlements cannot be overemphasized.
\end{abstract}

Key-words: Chagas disease. Infestation by triatomines. Rural settlements. Prevention and control.

A doença de Chagas é uma das afecções de mais ampla distribuição no continente americano. No Brasil, são mais de 100 espécies vetoras passíveis de transmitir a infecção natural por Trypanosoma cruzi, intervindo diretamente na sua veiculação no ambiente domiciliar ou participando na manutenção da enzootia chagásica ${ }^{23}$. Endêmica no Estado de São Paulo, a infecção chagásica tem atualmente, como principais espécies vetoras, Triatoma sordida, Panstrongylus megistus, Rhodnius neglectus e Triatoma tibiamaculata, as mais freqüentemente observadas pelo serviço de controle tanto durante busca ativa, quanto por envio, a nosso Serviço, de exemplares capturados por iniciativa de particulares ${ }^{15}$. Em consequiência do controle de populações de Triatoma infestans, principal vetor da doença de Chagas Estado de São Paulo até a década de 1970, outras espécies vetoras passaram a adquirir maior importância relativa ${ }^{15}$. Seus habitats silvestres e peridomiciliares distribuem-se pelo Estado de acordo com a feição paisagística de cada área ${ }^{6}$.

A história da doença de Chagas no Estado de São Paulo mostra que o homem, em suas relações com sua própria espécie e com o ambiente, estabeleceu condições favoráveis à disseminação de Triatoma infestans, a mais domiciliar de todas as espécies vetoras $^{17}$. A deterioração da qualidade de vida do homem ${ }^{23}$,

\footnotetext{
1. Laboratório de Imunoepidemiologia, Superintendência de Controle de Endemias, São Paulo, SP. 2. Serviço Regional de Presidente Prudente, Presidente Prudente, SP. 3. Laboratório de Parasitoses por Flagelados, Superintendência de Controle de Endemias, Mogi Guaçu, SP

Endereço para correspondência: Dr. Rubens Antonio da Silva. Laboratório de Imunoepidemiologia/SUCEN. R. Paula Sousa 166/5² andar, Luz, 01027-000 São Paulo, SP. e-mail: rubens@sucen.sp.gov.br

Recebido para publicação em 10/07/2006

Aceito em: 22/08/2007
} 
decorrente de problemas econômicos, exerceu forte influência na distribuição social dessa endemia. Com a consolidação das ações de controle, voltadas à principal espécie vetora, a vigilância entomológica e a participação da população na deteç̧ão de triatomíneos em seus domicílios foram implementadas em todo o Estado.

Necessidade de terra, de que resultaram reformas agrárias por um lado e empreendimentos hidrelétricos rurais por outro, provocaram a formação de assentamentos e reassentamentos rurais $^{19}$. Em primeiro lugar, o Movimento dos Trabalhadores Rurais Sem Terra (MST), levou os trabalhadores rurais a organizarem-se em busca de terras onde tivessem melhores condições de trabalho e qualidade vida ${ }^{11}$. Em segundo lugar, com o objetivo de reduzir os impactos ambientais originários dos empreendimentos hidrelétricos, a Companhia Energética do Estado de São Paulo (CESP) selecionou áreas de remanejamento de pequenos produtores rurais e suas famílias. Distribuídos por todo o Estado de São Paulo, o número desses novos agrupamentos aumentou gradativamente ao longo da década de $1990^{10}$, concentrando-se na região de Presidente Prudente ou, mais especificamente, no Pontal do Paranapanema (Figura 1). Baixa densidade demográfica e a existência de muitas terras devolutas constituíram estímulo para um processo da reforma agrária com a conseqüente distribuição de propriedades. Em busca de trabalho, famílias provenientes de diversas regiões do país deslocaram-se para essa área ${ }^{14}$, construindo novas unidades domiciliares (UD's), das quais destacamos, para efeito de pesquisa, intradomicílio e peridomicílio, este último constituído por anexos externos. A construção ulterior de anexos tende a fornecer novas fontes de alimento para triatomíneos. As moradias são de boa qualidade, sendo construídas de tijolos rebocados, mais expressivas nos reassentamentos rurais $(87,4 \%)$, constituindo-se também de casas de madeira, encontradas, principalmente nos assentamentos $(74,1 \%)$.

Na região de Presidente Prudente, já foi assinalada a presença de espécies de triatomíneos vetores da doença de Chagas, principalmente Triatoma sordida e Rhodnius neglectus ${ }^{15}$. Agressões ao ambiente podem reduzir ou mesmo eliminar as fontes de alimentação dos triatomíneos silvestres nativos e favorecer a colonização do domicílio humano. Mesmo em áreas consideradas endêmicas para a doença de Chagas, com vetores domiciliados, está presente o risco de a habitação humana ser colonizada por espécies nativas de triatomíneos ${ }^{21}$. Disto resulta a criação de novos focos de difusão da doença de Chagas humana ${ }^{23}$.

0 objetivo deste estudo foi o de avaliar a infestação, por triatomíneos, nos assentamentos e reassentamentos rurais levandose em consideração seu tempo de formação, de modo a subsidiar a proposição de ações de controle dirigidas a essas áreas.

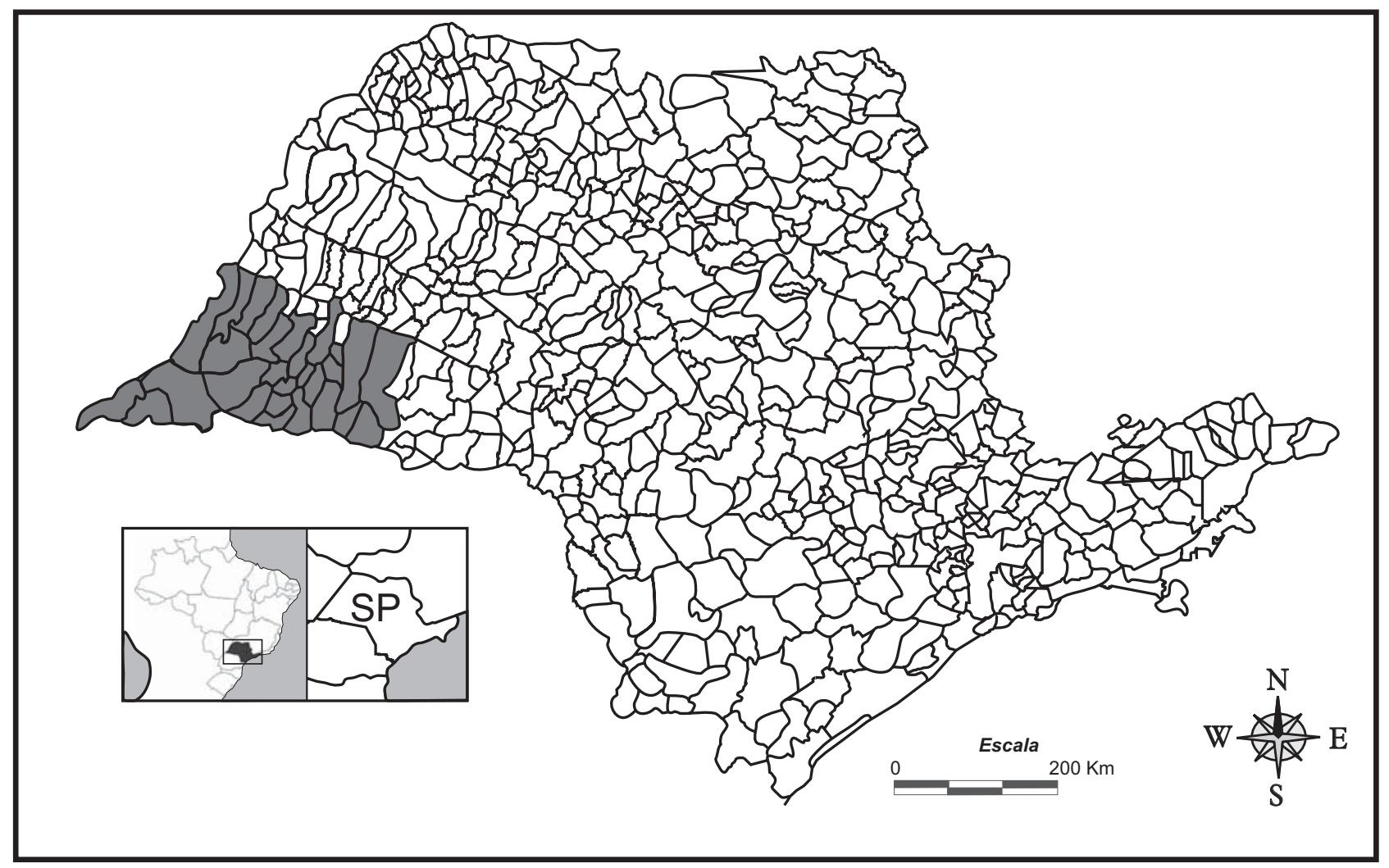

Figura 1 - Municípios que compõem o Pontal do Paranapanema. Estado de São Paulo, 2006. 


\section{MATERIAL E MÉTODOS}

O Pontal do Paranapanema está inserido na Região Administrativa de Saúde de Presidente Prudente, situada a sudoeste do Estado de São Paulo, Brasil. Compreende os municípios de Álvares Machado, Anhumas, Caiuá, Estrela do Norte, Euclides da Cunha Paulista, Iêpe, Indiana, Marabá Paulista, Martinópolis, Mirante do Paranapanema, Nantes, Narandiba, Piquerobi, Pirapozinho, Presidente Bernardes, Presidente Epitácio, Presidente Prudente, Presidente Venceslau, Rancharia, Regente Feijó, Rosana, Sandovalina, Santo Anastácio, Taciba, Tarabaí e Teodoro Sampaio. Ao todo, 25 municípios (Figura 1). A principal cidade dessa região, também conhecida como Alta Sorocabana, é Presidente Prudente, pólo regional da maior área pecuarista de São Paulo. Aí as pastagens se alternam com áreas de cerrado, com vários graus de devastação ${ }^{1}$. De acordo com 0 Instituto de Pesquisas Tecnológicas ${ }^{9}$, essa região está no Planalto Ocidental com formas de relevo, na sua maioria, de colinas amplas e médias.

0 clima da região caracteriza-se pela alternância de períodos secos e frios (entre maio e agosto, média de $17^{\circ} \mathrm{C}$ em junho) com os quentes e úmidos (entre novembro e fevereiro, média de $25^{\circ} \mathrm{C}$ ). Como extremos, podem verificar-se valores mínimos de até $-1,4^{\circ} \mathrm{C}$, (julho de 1981 ) e máximas de $38^{\circ} \mathrm{C}$ (fevereiro). As chuvas concentram-se nos meses de dezembro e janeiro. A precipitação pluvial média anual varia de $1.200 \mathrm{~mm}$ a $1.400 \mathrm{~mm}^{9}$.

Nesta avaliação, foram trabalhados os resultados de pesquisas de triatomíneos do período de janeiro de 1984 a junho de 2005, realizadas pelas equipes de campo da Superintendência de Controle de Endemias (SUCEN) por meio de ações preconizadas ${ }^{15}$, que incluíram busca ativa de triatomíneos, com pesquisa minuciosa nas unidades domiciliares dirigidas às fontes de alimentação, bem como no atendimento de notificações de insetos vetores pelos moradores dos assentamentos e reassentamentos rurais da região do Pontal do Paranapanema. Realizou-se controle químico nos locais de encontro de triatomíneos. Estes dados foram buscados em boletins de campo padronizados que continham informações sobre a presença de triatomíneos de acordo com o local de captura (intradomicílio e peridomicílio), identificação e fase evolutiva da espécie e presença de anexos.

Resultados de levantamentos com os números totais de assentamentos rurais e datas de sua formação foram fornecidos pela Fundação Instituto de Terras do Estado de São Paulo (ITESP), órgão responsável pelo planejamento e execução das políticas agrária e fundiária do Estado de São Paulo ${ }^{10}$. Dados sobre os reassentamentos rurais foram fornecidos pela Duke Energy Brasil, responsável pelo gerenciamento das usinas hidrelétricas da região e do acervo de dados 5 .

De acordo com suas datas de formação, os agrupamentos foram divididos em duas categorias: a primeira, dos formados com antecedência de menos de oito anos; a outra, dos formados com antecedência de oito anos ou mais, adotando-se como critério de corte, o tempo médio de formação. Os dados levantados foram expressos em termos de indicadores entomológicos e de valores relativos para assentamentos e reassentamentos: índice de infestação de casas = número de UD's positivas/número de casas pesquisadas $\times 100$; taxa de colonização $=$ número de ninfas coletadas no intradomicílio/número de triatomíneos coletados no intradomicílio $\times 100^{20}$ e taxa de aglomeração $=$ número de exemplares capturados/número de UD's infestadas $\times 100$, sendo este último indicativo da concentração de exemplares nos domicílios. As informações foram transcritas em banco de dados e as frequiências extraídas com o uso do programa Epiinfo versão 6.04 .

\section{RESULTADOS}

Durante o período de janeiro de 1984 a junho de 2005, foram verificados, no Estado de São Paulo, 165 assentamentos rurais ligados ao MST e seis reassentamentos, totalizando 171 agrupamentos. Os assentamentos distribuíram-se em 52 municípios do Estado, concentrando-se na região do Pontal do Paranapanema (Figura 2). Nessa região, foram assinalados $64,9 \%$ dos agrupamentos, sendo 105 assentamentos rurais e seis reassentamentos, compreendendo 18 (72\%) municípios.

0 tempo médio de formação dos agrupamentos foi de 7,4 anos (mediana $=7$ anos). Dos assentamentos, 71,4\% apresentavam idade de formação menor que oito anos, enquanto que nos reassentamentos este percentual foi de 33,3 (Tabela 1). Observouse que no total dos agrupamentos, $69,4 \%$ apresentaram tempo de formação menor do que oito anos.

No período em questão, foram realizadas 48 pesquisas de triatomíneos. As pesquisas realizaram-se integralmente em todas as UD's, através de busca ativa, pelas equipes de campo da SUCEN, em nove agrupamentos, sendo três caracterizados como assentamentos rurais e seis como reassentamentos. Os moradores, através da notificação de triatomíneos, foram responsáveis pelo desencadeamento da pesquisa em outras 25 habitações, sendo $10 \mathrm{em}$ assentamentos rurais e 15 em reassentamentos. Em 14 moradias, houve pesquisa de triatomíneos devido à positividade observada após o controle químico.

Nos assentamentos, foram realizadas 16 pesquisas de triatomíneos com sete $(43,7 \%)$ positivas, observadas nos de idade de formação maior ou igual a oito anos (Tabela 2). Já nos reassentamentos totalizaram-se 32 pesquisas resultando $71,8 \%$ positivas. Esta positividade foi mais $(78,5 \%)$ expressiva naqueles com tempo de formação menor que oito anos $\left(\chi^{2}=43,067 \mathrm{p}<0,001\right)$. A relação de anexos peridomiciliares, nos agrupamentos com pesquisa integral foi em média de 3/1 quando comparados reassentamentos e assentamentos.

A Tabela 3 mostra que foram coletados no período 795 exemplares de triatomíneos, sendo pertencentes às espécies Triatoma sordida $(98,9 \%)$ e Rhodnius neglectus $(1,1 \%)$, encontrados principalmente, em peridomicílios (97,5\%). A presença de triatomíneos foi mais freqüente nos reassentamentos do que nos assentamentos. Das coletas de exemplares adultos de triatomíneos nos intradomicílios de assentamentos, 23,5\% resultaram de atividades de notificação por moradores. 0 cálculo de indicadores entomológicos revelou $11,1 \%$ de 


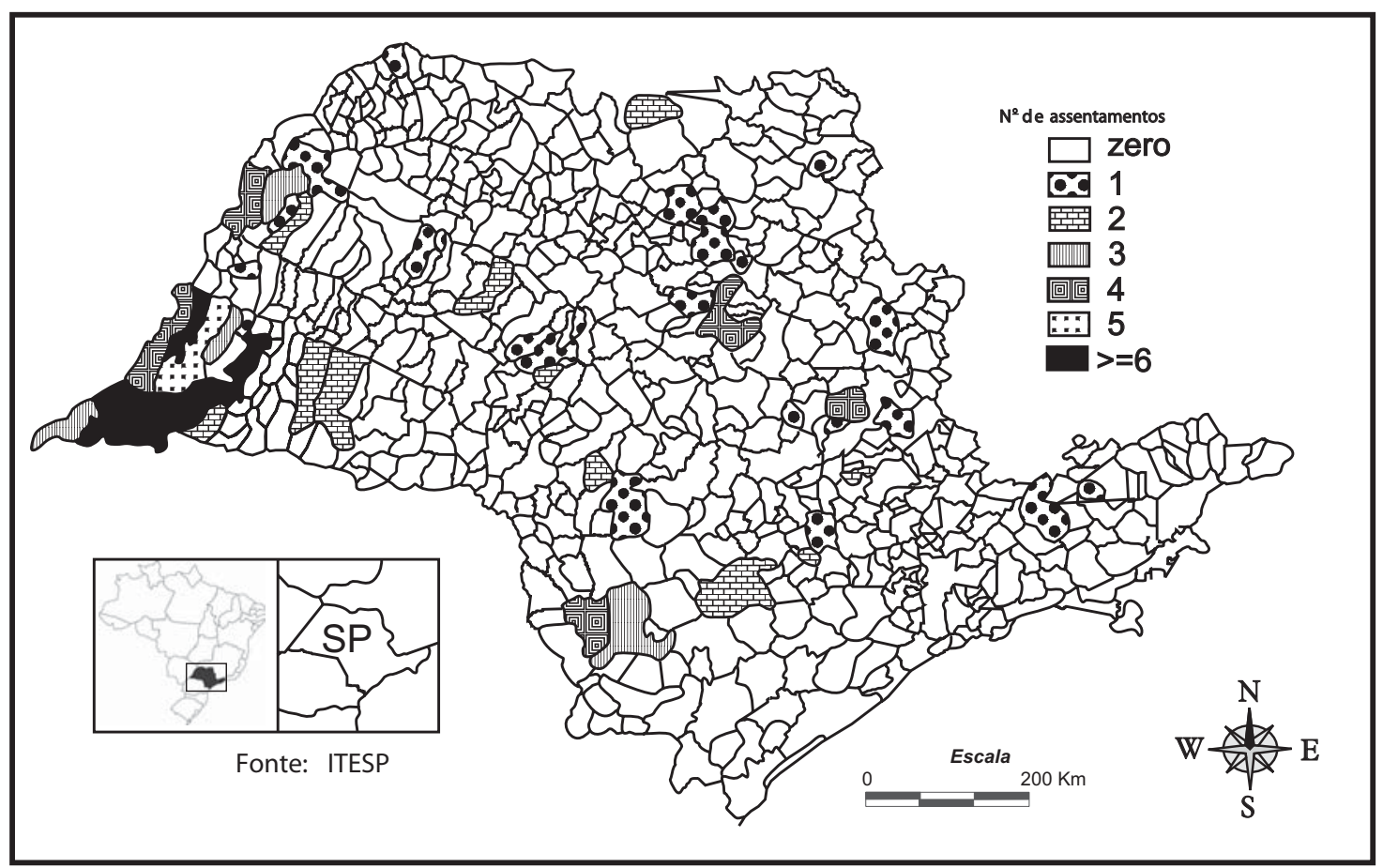

Figura 2 - Distribuição de assentamentos rurais segundo município: Estado de São Paulo, janeiro de 1984 a junbo de 2005.

Tabela 1 - Assentamentos e reassentamentos rurais segundo tempo de formação. Região do Pontal do Paranapanema, Estado de São Paulo, janeiro de 1984 a junbo de 2005.

\begin{tabular}{|c|c|c|c|c|c|c|}
\hline \multirow{3}{*}{ Agrupamento } & \multicolumn{6}{|c|}{ Tempo de formação (anos) } \\
\hline & \multicolumn{2}{|c|}{$<8$} & \multicolumn{2}{|c|}{$\geq 8$} & \multicolumn{2}{|c|}{ total } \\
\hline & $\mathrm{n}^{0}$ & $\%$ & $\mathrm{n}^{0}$ & $\%$ & $\mathrm{n}^{0}$ & $\%$ \\
\hline Assentamento & 75 & 71,4 & 30 & 28,6 & 105 & 100,0 \\
\hline Reassentamento & 2 & 33,3 & 4 & 66,7 & 6 & 100,0 \\
\hline Total & 77 & 69,4 & 34 & 30,6 & 111 & 100,0 \\
\hline
\end{tabular}

Fonte: ITESP/Duke Energy (diferença entre agrupamentos teste $\chi^{2}=3,876 \mathrm{p}=0,04898$ )

Tabela 2 - Pesquisa de triatomineos em assentamentos e reassentamentos rurais segundo tempo de formação e resultado da pesquisa. Pontal do Paranapanema, Estado de São Paulo, janeiro de 1984 a junbo de 2005.

\begin{tabular}{|c|c|c|c|c|c|c|c|c|c|}
\hline \multirow{3}{*}{ Agrupamento } & \multicolumn{9}{|c|}{ Pesquisa de triatomíneos/Tempo de formação (anos) } \\
\hline & \multicolumn{3}{|c|}{$<8$} & \multicolumn{3}{|c|}{$\geq 8$} & \multicolumn{3}{|c|}{ total } \\
\hline & realizadas & positivas & $\%$ & realizadas & positivas & $\%$ & realizadas & positivas & $\%$ \\
\hline Assentamento & 3 & 0 & 0,0 & 13 & 7 & 53,8 & 16 & 7 & 43,7 \\
\hline Reassentamento & 28 & 22 & 78,5 & 4 & 1 & 25,0 & 32 & 23 & 71,8 \\
\hline Total & 31 & 22 & 70,9 & 17 & 8 & 47,0 & 48 & 30 & 62,5 \\
\hline
\end{tabular}

(diferença entre agrupamentos, teste $\chi^{2}=43,067 \mathrm{p}<0,001$ )

Tabela 3 - Distribuição dos triatomíneos coletados segundo estádio evolutivo e local de captura nos assentamentos e reassentamentos rurais. Região do Pontal do Paranapanema, Estado de São Paulo, janeiro de 1984 a junbo de 2005.

\begin{tabular}{|c|c|c|c|c|c|c|c|}
\hline \multirow{3}{*}{ Agrupamento } & \multirow{3}{*}{$\begin{array}{l}\text { Estádio } \\
\text { evolutivo }\end{array}$} & \multicolumn{6}{|c|}{ Local de captura } \\
\hline & & \multicolumn{2}{|c|}{ intra } & \multicolumn{2}{|c|}{ peri } & \multicolumn{2}{|c|}{ total } \\
\hline & & $\mathrm{n}^{\underline{0}}$ & $\%$ & $\mathrm{n}^{\underline{0}}$ & $\%$ & $\mathrm{n}^{0}$ & $\%$ \\
\hline \multirow[t]{3}{*}{ Assentamento } & adultos & 8 & 23,5 & 26 & 76,4 & 34 & 29,8 \\
\hline & ninfas & 2 & 2,5 & 78 & 97,5 & 80 & 70,2 \\
\hline & subtotal & 10 & 8,8 & 104 & 91,2 & 114 & 100,0 \\
\hline \multirow[t]{3}{*}{ Reassentamento } & adultos & 4 & 3,6 & 110 & 96,4 & 114 & 16,7 \\
\hline & ninfas & 6 & 1,0 & 561 & 99,0 & 567 & 83,3 \\
\hline & subtotal & 10 & 1,5 & 671 & 98,5 & 681 & 100,0 \\
\hline Total & 20 & 2,5 & 775 & 97,5 & 795 & 100,0 & \\
\hline
\end{tabular}

intra: intradomicílio peri: peridomicílio (diferença entre agrupamentos, teste $\chi^{2}=66,589 \mathrm{p}<0,001$ ) 
infestação nos assentamentos e 31,9\% nos reassentamentos $\left(\chi^{2}=66,589 \mathrm{p}<0,001\right)$. Valores da taxa de colonização: $20 \%$ nos assentamentos e $60 \%$ nos reassentamentos. Valores da taxa de aglomeração, representada pelo número de exemplares de triatomíneos capturados nos domicílios infestados: 1.628,5\% nos assentamentos e de $862 \%$ nos reassentamentos. 0 exame de positividade para verificação de infecção por Trypanosoma cruzi revelou resultado negativo para todos os exemplares.

\section{DISCUSSÃO}

Estas avaliações apresentam dificuldades metodológicas comuns aos estudos que se valem de dados secundários; mesmo assim, justificam-se como primeiros passos de avaliação operativa da vigilância e análise da tendência de domiciliação de espécies de triatomíneos nestes agrupamentos.

O Governo do Estado de São Paulo desenvolve como política agrária diferenciada, a utilização de terras públicas, que já responde por mais da metade dos assentamentos rurais paulistas. São realizadas benfeitorias na propriedade, como a construção da moradia e anexos, como pocilgas, estábulos e currais. Em sua maioria, os projetos, que atualmente abrigam mais de 5.457 famílias ${ }^{10}$ (Figura 2), concentram-se no extremo-oeste paulista.

Como se sabe, a doença de Chagas ocorre tipicamente em ambientes rurais onde o Trypanosoma cruzi circula entre animais silvestres, peridomésticos, domésticos e o homem. Diversas espécies de vetores são responsáveis por sua transmissã $0^{21}$. Modificações ambientais de causa antrópica geram profundas mudanças fauno-florísticas e abrem novos espaços em áreas de colonização recente, o que favorece a invasão por triatomíneos ${ }^{2}$.

A espécie mais frequientemente detectada no ambiente domiciliar por moradores e pelos desinsetizadores, que trabalharam na área estudada através, principalmente da atividade de atendimento às notificações de triatomíneos por parte da população foi Triatoma sordida que, segundo Silva e $\mathrm{col}^{18}$, no Estado de São Paulo, têm sido freqüentemente capturada em domicílios. Normalmente, o encontro de insetos nas UD's está associado à falta de higiene, à desordem intradomiciliar e à presença de animais dentro das habitações, condições que favorecem a persistência de triatomíneos nas áreas rurais ${ }^{8}$. No Estado de São Paulo, o hábito do morador não cuidar do peridomicílio em áreas com persistência de infestação, pode ser incriminado como propício para a presença de triatomíneos ${ }^{18}$. 0 Triatoma sordida tem grande capacidade de estabelecer colônias em ecótopos artificiais. Alterações do ambiente natural parecem associar-se a altas densidades do Triatoma sordida ${ }^{7}$. Aliado a este fato, o controle químico, empregado apenas em locais do domicílio em que se constatou a presença de triatomíneos ${ }^{14}$ torna muito difícil obter um impacto sobre as espécies que não colonizam exclusivamente o intradomicílio. Além disto, os inseticidas não funcionam bem no peridomicílio ${ }^{12}$. Neste estudo, observamos a presença de colônias de Triatoma sordida no peridomicílio, o que pode sinalizar, segundo Diotaiuti e cols ${ }^{4}$, uma iminente colonização por esta espécie, caso as medidas de controle sejam descontinuadas. 0 Triatoma sordida possui grande resistência ao jejum e é eclético quanto à alimentação; pode ter maiores oportunidades para a conquista de número cada vez maior de ecótopos ${ }^{7}$. 0 manejo da pecuária, observado na área estudada, é um importante fator de risco para a infestação de triatomíneos conforme relatado por Walter e $\mathrm{col}^{24}$. Esse risco é em parte reduzido pelo próprio processo de adaptação dos triatomíneos à UD, que é lento e requer simplificações genéticas, biomorfológicas e de comportamento $0^{13}$. 0 maior número de exemplares de triatomíneos foi coletado nos reassentamentos rurais, observando-se nestes os maiores valores percentuais de infestação de casas e de colonização pelos insetos. A concentração de triatomíneos, entretanto, foi mais expressiva nos assentamentos rurais. 0 encontro de reassentamentos rurais com pesquisa de triatomíneos positiva pode estar relacionado com o transporte passivo desses insetos a partir de seu local de origem para este novo ambiente. Foi dada permissão aos moradores para retirarem da UD todos os seus pertences e transportá-los para a nova área, incorporando maior quantidade de anexos. Triatoma sordida pode ter dispersão passiva quando insetos desta espécie são transportados para ambientes artificiais junto com lenha, pelo homem, ou por pássaros ${ }^{3}$. Podem também se dispersar ativamente; pertencem a uma das espécies com maior capacidade de vô $0^{22}$. Nos assentamentos, a pequena quantidade de anexos domiciliares observada pode ser a razão da não presença de colônias. A positividade foi mais importante onde a formação era mais antiga e houve maior oportunidade para a incorporação de anexos, representados principalmente por galinheiros, onde fontes de alimento acessíveis tornam fácil a colonização por triatomíneos (Tabelas 2 e 3).

É certo que a transmissão natural da doença de Chagas no país foi grandemente reduzida e que há tecnologia bastante para sustentar os níveis de controle alcançados ${ }^{23}$. No intradomicílio, os resultados indicam que o encontro de triatomíneos tem ocorrido de forma ocasional: pequena densidade de triatomíneos resultantes de migração ativa a partir do ambiente silvestre. As estratégias de vigilância nos assentamentos com maior idade de formação devem ser implementadas, buscando-se maior envolvimento da população na detecção de insetos vetores. Como estes grupos humanos estão em constantes deslocamentos, tornase importante manter vigilância permanente, exercida pelo serviço de controle, a fim de descobrir qualquer possível instalação desses insetos em novas áreas. Trabalhos continuados de educação em saúde, visando ao conhecimento da doença e seus vetores, além da participação comunitária ativa devem ser implementados. Tem sido possível manter níveis de infestação e de colonização intradomiciliar incompatíveis com a transmissão no Estado por meio de um trabalho de vigilância de caráter contínuo, com pronta intervenção sempre que há evidência de constituição de colônias em domicílios. Hoje, a criação do Programa de Agentes Comunitários (PAC's) fornece meios que facilitam os contatos com a população local. Se bem explorado, este pode ser um caminho de grande valia.

Estudo realizado por Silva e cols ${ }^{19}$, em assentamentos e reassentamentos rurais do Estado de São Paulo, sobre 
conhecimentos a respeito da doença de Chagas e seus vetores por moradores, permitiu verificar que, em assentamentos houve menor percentual de pessoas que reconheciam os vetores e tinham informações sobre a doença, ao contrário do que ocorria nos reassentamentos, caracterizados na época do estudo pelo fato de abrigarem pessoas nativas do Estado. Certas enfermidades têm maior significado para as comunidades do que outras, devido às suas características epidemiológicas.

0 desafio consiste em repensar a noção de participação com experiências inovadoras, simultaneamente às atividades de cunho tradicional já existentes. É possível vivenciar a diversidade cultural, contribuir com a construção democrática e ampliação da idéia de cidadania como garantia e conquista. É fundamental que o espaço formal da educação seja envolvido no processo de vigilância epidemiológica contra a doença de Chagas ${ }^{2}$. A municipalização das ações educativas do Programa de Controle da Doença de Chagas, desenvolvido no Estado de São Paulo, foi posta em efeito a partir do ano de 2004. Em um primeiro momento esta participação se mostrou profícua. 0 componente educativo tem-se revelado imprescindível para a elaboração de estratégias de ações participativas e de comunicação para que a população se aproprie do programa e se comprometa a participar do controle e da vigilância entomológica de triatomíneos em seu meio. Para a sustentabilidade das ações, deve-se manter atualização técnica das equipes multiprofissionais envolvidas nas atividades do Programa. Orientações da população quanto à vigilância e ao controle de triatomíneos dentro de uma óptica realística e de maior impacto devem propiciar diálogo e, através de uma relação democrática com a população local, é gerada uma participação nas decisões, fortalecendo-se a conquista coletiva de prática de estilos de vida saudáveis, especialmente no combate à doença.

\section{AGRADECIMENTO}

Ao Prof. Cláudio dos Santos Ferreira, pela revisão crítica do manuscrito.

\section{REFERÊNCIAS}

1. Baptista FAN. Breve histórico do Pontal do Paranapanema. http://members.tripod. com/piquerobi/breve_historico_do_pontal_do_paranapanema.htm. Acessado em 18/07/2004, 2003.

2. Dias JCP. Vigilância epidemiológica em doença de Chagas. Cadernos de Saúde Pública 16 (supl 2): 43-59, 2000.

3. Diotaiuti L. A situação epidemiológica do Triatoma sordida após o controle do Triatoma infestans. Revista da Sociedade Brasileira de Medicina Tropical 30 (sup I): 54-56, 1997.

4. Diotaiuti L, Paula OR, Falcão PL, Dias JCP. Evaluation of the Chagas disease vector control program in Minas Gerais, Brazil with special reference to Triatoma sordida. Bulletin of the Pan American Health Organization 28:211-219, 1994.

5. Duke Energy Brasil. Usinas. Fundação Instituto de Terras do Estado de São Paulo "José Gomes da Silva". (http://www.duke-energy.com.br/PT/Usinas/index.asp). Acessado em 28/03/2006), São Paulo, 2006.
6. Forattini OP. Biogeografia, origem e distribuição da domiciliação de triatomíneos no Brasil. Revista de Saúde Pública 14:265-299, 1980.

7. Forattini OP, Ferreira AO, Silva EOR, Rabello EX. Aspectos ecológicos da tripanossomíase americana. V. Observações sobre colonização espontânea de triatomíneos silvestres em ecótopos artificiais, com especial referência ao Triatoma sordida. Revista de Saúde Pública 7:219-239, 1973.

8. Garcia-Zapata MTA, Marsdsen PD. Enfermedad de Chagas: control y vigilancia con insecticidas y participación comunitaria en Manabí, Goiás, Brasil. Boletim da Oficina Sanitária Panamericana 116: 97-110, 1994.

9. Instituto de Pesquisas Tecnológicas do Estado de São Paulo (IPT). Mapa Geomorfológico do Estado de São Paulo, volume I e II, mapa escala 1:500.000, 1981

10. Instituto de Terras do Estado de São Paulo "José Gomes da Silva" (ITESP). Assentamentos história em São Paulo. Site: http://www.itesp.sp.gov.br/acprogres/ resultados/tabela.htm). Acessado em 28/03/2006), 2006

11. Movimento dos Trabalhadores Rurais Sem Terra (MST). A luta do MST e a questão agrária do Estado de São Paulo. (http://www.mst.org.br/mstsp/hist. html). Acessado em 28/03/2006), 2000.

12. Oliveira-Lima JW, Faria Filho OF, Vieira JBF, Gadelha FV, Oliveira Filho AM. Alterações do peridomicílio e suas implicações para o controle do Triatoma brasiliensis. Cadernos de Saúde Pública 16 (supl 2): 75-81, 2000.

13. Schofield CJ, Dias JCP. The southen cone programme against Chagas disease. Advances in Parasitology 42: 1-25, 1999.

14. Secretaria de Estado da Justiça e Defesa da Cidadania (SJDC). Retrato da Terra: Perfil sócio-econômico dos assentamentos do Estado de São Paulo - 96/97. Instituto de Terras do Estado de São Paulo "José Gomes da Silva", Secretaria de Estado da Justiça e Defesa da Cidadania, 1998.

15. Secretaria de Estado da Saúde do Estado de São Paulo (SES). Relatório do Grupo de Trabalho, Revisão do Programa de Controle da Doença de Chagas, São Paulo. Superintendência de Controle de Endemias, 2002.

16. Silva EOR, Wanderley DMV, Rodrigues VLCC. Triatoma infestans: importância, controle e eliminação da espécie no Estado de São Paulo, Brasil. Revista da Sociedade Brasileira de Medicina Tropical 31: 73-88, 1998.

17. Silva LJ. A evolução da doença de Chagas no Estado de São Paulo. Editora Hucitec São Paulo, 1999.

18. Silva RA, Rodrigues VLCC, Carvalho ME, Pauliquévis-Júnior C. Programa de Controle da Doença de Chagas no Estado de São Paulo: persistência de infestação por triatomíneos em localidades na década de 1990. Cadernos de Saúde Pública 19:965-971, 2003.

19. Silva RA, Sampaio SMP, Poloni M, Koyanagui PH, Carvalho ME, Rodrigues VLCC. Pesquisa sistemática positiva e relação com conhecimento da população de assentamento e reassentamento de ocupação recente em área de Triatoma sordida (Hemiptera, Reduviidae) no Estado de São Paulo, Brasil. Cadernos de Saúde Pública 20:555-561, 2004.

20. Silveira AC. Indicadores operacionais para um programa de eliminação do Triatoma infestans. Revista da Sociedade Brasileira de Medicina Tropical 26 (supl III): 51-54, 1993.

21. Silveira AC, Costa JR. Doença de Chagas e controle do vetor: desafios atuais, habitacionais e ambientais. Revista de Patologia Tropical 29 (supl I): 199-212, 2000.

22. Soares RPP, Pires HHR, Diotaiuti L. Estudos sobre a capacidade de vôo do Triatoma sordida sob condições de laboratório. Revista da Sociedade Brasileira de Medicina Tropical 29 (supl 3): 84-85, 1996.

23. Vinhaes MC, Dias JCP. Doença de Chagas no Brasil. Cadernos de Saúde Pública 16 (supl 2):7-12, 2000.

24. Walter A, Rego IP, Ferreira AJ, Rogier C. Risk factors for reinvasion of human dwellings by sylvatic triatomines in northern Bahia State, Brazil. Cadernos de Saúde Pública 21: 974-978, 2005. 